contains polylactosamine repeats representing potential ligands for animal lectins called galectins, implicated in HIV pathogenesis.

Methods CPI-GC was isolated from $T$. vaginalis LPG by mild acid hydrolysis and C18-SepPak separation. Binding to galectin-1 and -3 (Gal-1 and -3) was determined by Biolayer Interferometry. Inflammation-related proteins and Gal-1 and 3 were measured by a multiplex immunoassay in supernatants from human cervical and vaginal epithelial cells infected with T. vaginalis or exposed to CPI-GC from different clinical isolates.

Results CPI-GC activated NF- $\kappa B$ and upregulated cFos, COX-2, IL-8, MIP-3 $\alpha$, IL-6, IL-1 $\beta$ and VEGF in a MEK1/2 dependent manner. In addition, IL- 6 , ICAM-1 and VEGF up-regulation was mediated by p38 while IL- 8 and MIP- $3 \alpha$ were ERK $1 / 2$ mediated. CPI-GC from different clinical isolates varied in their ability to bind Gal-1 and Gal-3, which were constitutively expressed by vaginal and cervical epithelial cells and released at higher levels in the extracellular space during exposure to live trichomonas and CPIGC. CPI-GC from all isolates invariably reduced levels of the natural microbicide SLPI. Mutant trichomonads that failed to bind Gal-1 and Gal-3 showed higher proinflammatory activity suggesting a role for the CPI-GC -galectin binding in suppressing innate immune responses.

Conclusion Interventions targeting CPI-GC or restoring the balance of natural immune defences represent a promising strategy for preventing adverse outcomes from $T$. vaginalis infection.

\subsection{REGULATORY T CELLS IN PERIPHERAL BLOOD AND CEREBROSPINAL FLUID OF SYPHILIS PATIENTS WITH AND WITHOUT NEUROLOGICAL INVOLVEMENT: A COMPREHENSIVE AND COMPARATIVE STUDY}

doi:10.1136/sextrans-2013-051184.0085

LI K, C Wang, H Lu, X Gu, Z Guan, P Zhou. Shanghai Skin Disease Hospital, Shanghai, China

Background Syphilis, a sexually transmitted disease caused by spirochetal bacterium Treponema pallidum, can progress to affect central nervous system, causing neurosyphilis. While many neurosyphilis patients may be asymptomatic, some patients can develop severe neurological and psychiatric symptoms. Accumulating evidence suggest that skin lesions and clinical symptoms of early syphilis patients result from host immune and inflammatory responses. However, very little is known about the immune components in neurosyphilis.

Methodology/Principal Findings In the present study, we perform a comprehensive and comparative analysis of regulatory $\mathrm{T}$ cells (Tregs) between 102 neurosyphilis patients and 431 syphilis patients without neurological involvement. We found secondary and serofast patients had increased Treg percentage, suppressive function and TGF- $\beta$ levels in peripheral blood compared to healthy donors and serum Rapid Plasma Reagin (RPR) titers were positively correlated with Treg numbers in these patients. Neurosyphilis patients had higher Treg frequency in peripheral blood than those of syphilis patients without neurological involvement. Importantly, CD4+ T cells were increased and predominated in cerebrospinal fluid (CSF) of both asymptomatic and symptomatic neurosyphilis patients. Interestingly, a significant decrease in CSF CD4+ CD25 high Treg percentage was observed in symptomatic neurosyphilis patients compared to those of asymptomatic neurosyphilis patients, which may be associated with low CSF TGF- $\beta$ levels.

Conclusions Our findings suggest that neurological progression in syphilis patients may be associated with an enhanced systemic Treg response and an increased local CD4+ $\mathrm{T}$ cell infiltration. A decrease in Treg frequency in CSF of symptomatic neurosyphilis patients indicates that immune-mediate tissue damage might be involved in the development of neurological symptoms.

\section{O01.4 BLOOD TRANSCRIPTIONAL PROFILING OF WOMEN WITH CHLAMYDIA TRACHOMATIS IDENTIFIES A PELVIC INFLAMMATORY DISEASE (PID) SIGNATURE}

\author{
doi:10.1136/sextrans-2013-051184.0086
}

T Darville, X Zheng, C O'Connell, U Nagarajan, I Macio, H Wiesenfeld, L Rabe, S Hillier. University of Pittsburgh, Pittsburgh, PA, United States

Objective Most women with Chlamydia trachomatis (CT) infection are asymptomatic, while $\sim 3 \%$ progress to pelvic inflammatory disease (PID) within two weeks of untreated infection. The identification of biomarkers that predict development of PID would aid in identification of women at risk for complications of infertility and ectopic pregnancy. The specific aim of this study was to identify a whole blood transcript signature for acute PID due to chlamydial infection.

Methods We performed gene expression microarrays using whole blood from 79 women who had a gynecologic exam, and cervical and endometrial microbiologic testing. Samples were divided into five groups: Group 1, women with acute PID who were CT+ at endometrium (PID+, CT+, and $\mathrm{E}+$ ); Group 2, asymptomatic women who were CT + at endometrium (PID-, CT +, E+); Group 3 , asymptomatic women who were CT + at cervix (PID-, CT +, E-); Group 4, asymptomatic women who were CT- at cervix and endometrium (PID-, CT-, E-); Group 5, women with symptoms of PID who were negative for CT or other sexually transmitted pathogens (PID+, STI-, E-).

Results We identified a transcript signature that discriminated women with chlamydial PID from all other groups. Pathway analysis revealed that the chlamydial PID signature contained genes from interferon response pathways. Gene transcription in a subset of women with chlamydial endometrial infection clustered with women with chlamydial PID.

Conclusions Our study raises the possibility that transcriptional biomarkers with potential as diagnostic and prognostic tools can be identified to combat chlamydial reproductive tract disease in women

\subsection{EFFICACY OF RG1-VLP VACCINATION AGAINST GENITAL AND CUTANEOUS HUMAN PAPILLOMAVIRUSES IN VITRO AND IN VIVO}

doi:10.1136/sextrans-2013-051184.0087

C Schellenbacher, ${ }^{2} \mathrm{~K}$ Kwak, ${ }^{3} \mathrm{D}$ Fink, 'S Shafti-Keramat, 'B Huber, ${ }^{1} \mathrm{C}$ Jindra, ${ }^{2} \mathrm{R}$ Roden, ${ }^{1} \mathrm{R}$ Kirnbauer. 'Medical University Vienna, Division of Immunology, Allergy and Infectious Diseases (DIAID), Vienna, Austria; ${ }^{2} J o h n s$ Hopkins University, Baltimore, MD, United States; ${ }^{3} 3$ Institute of Laboratory Animal Science, Veterinary University Vienna, Austria, Vienna, Austria

Licensed human papillomavirus (HPV) vaccines, based on virus-like particles (VLP) self-assembled from major capsid protein L1, afford type-restricted protection against types 16/18/6/11 (or 16/18 for the bivalent vaccine), which cause $70 \%$ of cervical carcinomas (Cxca) and $90 \%$ of genital warts. However, they do not protect against less prevalent high-risk types causing $30 \%$ of $\mathrm{CxCa}$, or cutaneous HPV. The minor capsid protein $\mathrm{L} 2$ confers low-level immunity to type-common epitopes.

Chimeric RG1-VLP presenting HPV16L2 amino acids 17-36 (RG1 epitope) within the DE-surface loop of HPV16L1 induce crossneutralisation in vitro. We hypothesised, that RG1-VLP vaccination protects against a large spectrum of mucosal and cutaneous HPV infections in vivo.

L2-specific antibody and CTL responses in RG1-VLP vaccinated rabbits were determined by ELISA and ELISPOT assays. Cross-neutralisation was analysed using native or pseudovirion (PsV) assays. Vaccine efficacy in vivo was determined in a mouse genital challenge model. 